\title{
BMJ Open Quality Cardiac arrest in the paediatric intensive care unit: defining the problem and developing solutions
}

To cite: Ray CM, Pizzuto M, Reyes-Alvarado $\mathrm{E}$, et al. Cardiac arrest in the paediatric intensive care unit: defining the problem and developing solutions. BMJ Open Quality 2020;9:e000930. doi:10.1136/ bmjoq-2020-000930

- Additional material is published online only. To view, please visit the journal online (http://dx.doi.org/10.1136/ bmjoq-2020-000930).

Received 21 January 2020 Revised 10 July 2020 Accepted 29 November 2020

Check for updates

(c) Author(s) (or their employer(s)) 2020. Re-use permitted under CC BY-NC. No commercial re-use. See rights and permissions. Published by BMJ.

${ }^{1}$ Pediatric Critical Care, WakeMed Raleigh Campus, Raleigh, North Carolina, USA ${ }^{2}$ Pediatric Critical Care, University of North Carolina at Chapel Hill, Chapel Hill, North Carolina, USA

${ }^{3}$ Pediatric Critical Care, Duke University, Durham, North Carolina, USA

Correspondence to Dr Candice M Ray; cray@wakemed.org

\section{ABSTRACT}

Thousands of children experience a cardiac arrest event in the hospital each year, with more than half of these patients not surviving to hospital discharge. Cardiopulmonary resuscitation (CPR) depth, rate, velocity and percentage of high-quality chest compressions are modifiable factors associated with improved survival. Therefore, we created a novel and standardised process to track and analyse cardiac arrests in the Duke paediatric intensive care unit (PICU). Our aim was to identify areas for improved American Heart Association (AHA) compliance and implement education and communication-based initiatives to enhance early recognition of at-risk patients leading to improved outcomes. From January 2017 to December 2018, all cardiac arrests in our PICU were tracked, reviewed and presented at monthly morbidity and mortality conference. We used the data to track compliance with AHA guidelines and identify opportunities for improvement. Through these efforts, we established a multidisciplinary cardiac arrest education and review programme. Over the 2-year period, we tracked 45 cardiac arrests, which comprised $2 \%$ of all PICU admissions. In 2017, during the first year of development, 16 of 22 arrests $(73 \%)$ were not reported to code committee members in time for complete review. Of the six cardiac arrests with complete reviews, only $17 \%$ followed AHA guidelines. In 2018, all 23 arrest events were communicated and $76 \%$ of resuscitations were found to be compliant with AHA guidelines. Survival of patients to discharge was $47 \%$ in 2017 and increased to $63 \%$ in 2018 with similar percentage of PICU admissions having a cardiac arrest between the 2 years. The primary aim of this project was to establish a multidisciplinary comprehensive cardiac arrest review process. This programme allowed for comprehensive analysis of individual events, promoted quality improvement initiatives and improved consistent delivery of high-quality CPR.

\section{PROBLEM/BACKGROUND}

Approximately 6000 paediatric patients receive in-hospital cardiopulmonary resuscitation (CPR) each year, with $90 \%$ of these cardiac arrest events taking place in the paediatric intensive care unit (PICU) ${ }^{1-3}$ Patients who have cardiac arrests represent a significant proportion of paediatric mortality, with only $45 \%$ surviving to hospital discharge. ${ }^{1}$ High-quality CPR is associated with improved survival, ${ }^{3}$ however, CPR guidelines are often not met during resuscitation attempts. ${ }^{4}$

In an effort to improve compliance with American Heart Association (AHA) resuscitation guidelines, the AHA launched the 'Get with the Guidelines-Resuscitation' programme. Their goal was to create performance benchmarks and evidence-based feedback across all patient populations. ${ }^{5}$ As part of this effort, the AHA recommends specific parameters for CPR depth, rate, velocity and percentage of high-quality chest compressions. These are well studied modifiable factors that impact survival in patients who experience both in and out of hospital cardiac arrest. ${ }^{2}$ A retrospective cohort study from the AHA Get With The Guidelines-Resuscitation Registry showed that over $50 \%$ of adult cardiac arrests have deviations from recommended guidelines. Deviations from the AHA guidelines were associated with decreased rate of return of spontaneous circulation (ROSC) ${ }^{6}$ The use of resuscitation quality bundles like the CPR Coaching, Objective-Data Evaluation, Action-linked-phrases, Choreography, Ergonomics, Structured debriefing and Simulation (CODE ACES 2) have been associated with increased AHA CPR guideline compliance during in-hospital arrests. ${ }^{7}$ The use of multidisciplinary performance debriefing tools not only improves team performance, but improves patients short-term outcomes. ${ }^{8}$

There are a wide variety of approaches to improve the delivery of high-quality CPR, which include standard resuscitation courses, real time feedback devices and postarrest team debriefing tools. Standard resuscitation courses are the mainstay of CPR training and maintenance of certification, yet evidence is lacking that these courses improve outcomes. ${ }^{9} 10$ Courses of this nature have the issue of diminishing performance over time, with loss of skills as soon as 1 year after training. ${ }^{10}$ One approach to addressing this issue of skill loss over time 
is incorporating real time feedback via devices that are integrated into defibrillators and other devices, and these interventions have demonstrated moderate improvement with AHA guideline compliance. However, these systems focus strictly on improving technical aspects of basic life support performance. ${ }^{3}$

A more comprehensive approach to resuscitation education is integration of post-arrest team debriefing tools into reviews of resuscitation efforts. This approach has been demonstrated to improve CPR quality, shortterm survival and neurological outcomes in both adult and paediatric patients. ${ }^{3}$ A multidisciplinary approach in postarrest debriefings of this nature further enhances compliance with paediatric AHA guidelines and improves CPR outcomes. ${ }^{4}$ Debriefings can also help facilitate discussion after an event to improve future communication among the resuscitation team members. ${ }^{11-13}$ Debriefings also allow healthcare workers educational opportunities that are relevant, goal-oriented, practical and collaborative. ${ }^{11}$ Current evidence suggests that paediatric postcardiac arrest debriefing programmes are feasible and useful for providers, regardless of their participation in the resuscitation. ${ }^{8}$

Given the importance of high-quality CPR in improving patient outcomes, percentage of arrest resulting in ROSC and improved percentage of hospital discharges, we desired to study cardiac arrests in the Duke PICU to address the lack of a standardised system to track incidence, performance and outcomes of cardiac arrest through a comprehensive debriefing programme. Due to the lack of a tracking system or preintervention data we were unable to target specific areas for improvement during a cardiac arrest event nor were we able to track our cardiac arrest outcomes compared with published data. Therefore, we felt that it was critical to established a quality improvement programme around cardiac arrest events and CPR in our PICU.

\section{MEASUREMENTS}

The Duke PICU is part of Duke Children's Hospital $(\mathrm{DCH})$, which is a quaternary care centre with a diverse population and wide range of patient diagnoses. The PICU has over one thousand patients admitted annually, with a wide spectrum of medical and surgical diagnoses, including both solid organ and bone marrow transplant recipients. Acute postoperative patients with congenital heart disease are cared for in a separate paediatric cardiac intensive care unit. The facility has full Extracorporeal Membrane Oxygenation (ECMO) capabilities including ECMO-CPR (ECPR).

At DCH, the leader for cardiopulmonary resuscitative efforts is either the PICU fellow, advanced practice provider (APP) or a paediatric intensivist in both the PICU or in-patient floors. The PICU is staffed 24 hours a day, 7 days a week by a paediatric intensive care physician who completed an Accreditation Council for Graduate Medical Education accredited paediatric critical care fellowship who is board eligible or certified. These physicians have a range of experience as paediatric critical care physicians from 2 to 10 years. Thus, a paediatric intensivist is present for all cardiac arrest events. All PICU physicians, APPs, residents, PICU fellows, nurses and respiratory therapists are trained in PALS.

The code team for the PICU includes a paediatric intensivist, a paediatric intensive care fellow, a paediatric intensive care nurse practitioner, a respiratory therapist and 1-3 PICU nurses. There are three code carts in the PICU. Two code carts are the Paediatric Code Carts (Blue) that are made by ARMSTRONG MEDICAL (A-SMART CART SYSTEMS). Each Paediatric Blue Code Cart is checked each shift by the charge nurse to ensure the seal is intact. If the seal is broken, then a full cart check is done. The carts are checked monthly for expired products and medications. There is also one Universal Code Cart (Red) (MMP Medcart), which is standardised hospital-wide. This code cart is checked and restocked once a month. The Hospital Code Committee oversees contents and processes related to the Universal Code Cart (Red) while the Paediatric Code Blue Committee, which is a subcommittee of the Hospital Code Committee, oversees the Paediatric Code Carts (Blue). There are two defibrillators in the PICU (LIFEPAK 20e) and we use Physio Control Quick-Combo pads (paediatric and adult) for defibrillation,

A key driver diagram was created to inform our project and is included in online supplemental documents. From January 2017 to December 2018, all cardiac arrests in the PICU were tracked and reviewed in detail. A paediatric critical care fellow was notified when a patient received chest compressions of any duration in the PICU. Within 48 hours, the PICU fellow and faculty code champions performed a comprehensive review of the available data.

Each individual cardiac arrest was analysed and presented at a monthly multidisciplinary Morbidity and Mortality (M\&M) conference. Year-to-date data were aggregated and presented at the beginning of each calendar year. PICU faculty and staff members, graduate medical education trainees and consulting services attended each meeting regardless of their involvement with specific patients or resuscitation efforts.

At each session, a templated review of each arrest was presented. This template is included in online supplementary material. Each presentation started with the ground rules for participation with emphasis on the peer-protected nature of the discussion and importance of a professional and blame-free environment. Next, we addressed basic event information including time and date of event, duration of the arrest, time to CPR, quality of CPR including rate and depth, medications administered, rate of ROSC, need for ECPR and patient outcome. Patient information included: medical history, admission diagnosis, procedures, labs and prior pertinent imaging. Vital signs were tracked for the prior 12 hours to the event and throughout the entire resuscitation effort. We presented graphics of arrest data including vital signs, ECG with rate and rhythm, pulse oximetry tracing 
and arterial line waveform, non-invasive cardiac output monitor data if on invasive ventilatory support and nearinfrared spectroscopy trends. Code-specific information included prearrest rhythm, CPR interruption and rationale (pulse check, intubation attempt, ECMO cannula placement), defibrillation attempts with preshock and postshock rhythm and chest compression quality including rate and depth. We assessed compliance with AHA guidelines. Cardiac arrest events were considered compliant if they followed all the recommended AHA guidelines for CPR including: no pauses in CPR lasting longer than $10 \mathrm{~s}$, assessment of high quality compressions by analysis of the arterial wave form or pulse oximeter waveform, code medications given at appropriate paediatric dosing and intervals, shockable rhythms were treated at appropriate Joule dosing, and there was no greater than a 2 min delay in CPR once a patient met cardiac arrest criteria. We targeted areas for clinical care and CPR improvement including assessment of prearrest warning signs, delayed time to initiation of CPR, communication to and from the code leader and code documentation. Examples of high-quality CPR and resuscitative efforts and exceptional teamwork and communication were also highlighted during the reviews. Peer-to-Peer discussions and feedback were preformed after each cardiac arrest event with nursing, provider and respiratory therapist. These discussions were standardised and included a set of questions addressing: events leading up to the arrest, individual concerns about the patient prior to the arrest, were concerns addressed prior to arrest, what went well during the arrest, any equipment or medication issues, were code roles defined, was there closed loop communication, what did not go well and what could have been done differently in the same situation and finally additional constructive thoughts (table 1). Examples of templates for providers, respiratory therapists and nursing peer-topeer discussions are also included in online supplemental materials.

Project personnel entered data into Research Electronic Data Capture (REDCap) database hosted by Duke University. REDCap is a secure, web-based application designed exclusively to support data capture for research studies. Members of the research team completed all required trainings in the protection of human subjects. In this way, access to any patient identifiers was limited to the minimum necessary to carry out this project. All data and records generated were kept confidential in accordance with institutional policies on subject privacy and HIPPA regulations. This project was reviewed by the Duke IRB and granted IRB exemption. It was not appropriate or possible to involve patients or the public in the design, conduct, reporting or dissemination plans in this quality improvement initiative.

\section{DESIGN/STRATEGY}

With our first Plan-Do-Study-Act (PDSA) cycle, we set out to establish the creation of a multidisciplinary paediatric
Table 1 Cardiac arrest review components including core review topics, patient-specific information, prearrest and arrest data

\begin{tabular}{|c|c|}
\hline \multicolumn{2}{|l|}{$\begin{array}{l}\text { Cardiac arrest review } \\
\text { components }\end{array}$} \\
\hline \multicolumn{2}{|l|}{ Core review components } \\
\hline & Event time and date \\
\hline & Duration of cardiac arrest \\
\hline & Time to CPR \\
\hline & Quality of CPR \\
\hline & Depth, rate, $\geq 10$ s pauses \\
\hline & Time to ROSC \\
\hline & Time to ECMO \\
\hline & Patient outcome \\
\hline \multicolumn{2}{|c|}{ Patient demographic and clinical information } \\
\hline & Medical history \\
\hline & Admission diagnosis \\
\hline & Hospital procedures \\
\hline & Labs in the past 24 hours \\
\hline & Imaging in the past 24 hours \\
\hline & Vital signs from the prior 12 hours \\
\hline \multicolumn{2}{|l|}{ Precardiac arrest data } \\
\hline & ECG rate and rhythm \\
\hline & Pulse oximetry tracing \\
\hline & Arterial line wave form \\
\hline & NICO monitor data \\
\hline & NIRS trends \\
\hline \multicolumn{2}{|l|}{ Cardiac arrest data } \\
\hline & Prearrest rhythm \\
\hline & CPR interruption and rationale \\
\hline & $\begin{array}{l}\text { Pulse check, ECMO cannulation, intubation } \\
\text { attempt }\end{array}$ \\
\hline & Defibrillation attempts \\
\hline & Preshock and postshock attempts \\
\hline & $\begin{array}{l}\text { Chest compression quality including depth } \\
\text { and rate }\end{array}$ \\
\hline \multicolumn{2}{|l|}{ Peer-to-peer discussion } \\
\hline & Events leading up to the arrest \\
\hline & $\begin{array}{l}\text { Individual concerns about patient prior to } \\
\text { arrest, if so, were those concerns addressed }\end{array}$ \\
\hline & What went well during the arrest \\
\hline & Equipment or medication issues \\
\hline & Were code roles well defined \\
\hline & $\begin{array}{l}\text { What could have been done differently in the } \\
\text { same situation }\end{array}$ \\
\hline & Any additional constructive thoughts \\
\hline
\end{tabular}

CPR, cardiopulmonary resuscitation; ECMO, extracorporeal membrane oxygenation; NICO, non-invasive cardiac output; NIRS, near-infrared spectroscopy; ROSC, return of spontaneous circulation.

cardiac arrest review process. Information about the review programme was sent to faculty and staff by email and posters were placed in the PICU provider work areas. The development of a 'code corner' was created to house code sheets and debriefing documents in one 
easily visible centralised location. Two PICU fellows were trained on the code review process, including use of the central telemetry system. We found that despite education, awareness and a centralised location for arrestrelated forms, there were multiple missed code event notifications early in the process. After eliciting feedback during the cardiac arrest review meetings from nursing, PICU fellows and respiratory therapists, we noted many did not know how to notify the committee members of an arrest and were unaware of some code related documentation processes. During this time, we also held cardiac arrest reviews as an independent educational conference. However, subjectively minimal attendance with often less than ten people in attendance and little discussion about arrest events. Given our initial challenges, we sought to improve notification of cardiac arrests and to improve attendance at the cardiac arrest review meetings. Despite low attendance at review meetings, we were encouraged by the overall enthusiasm and buy-in from all invested participants to develop a standardised approach to the cardiac review process. We successfully completed nine cardiac arrest reviews during our first PDSA cycle that occurred between January 2017 and April 2017. However, although we did complete nine cardiac reviews, many of the reviews had missing information due to lack of timely notification.

To help improve notification and education around the cardiac arrest review process, our second PDSA cycle involved developing a multidisciplinary committee made up of PICU nurses, respiratory therapists, PICU fellows, paediatric intensivists, nursing leadership and respiratory leadership. This second PDSA cycle spanned from April 2017 to July 2017. This committee was responsible for notifying the PICU fellow of recent arrests and helping staff to fill out appropriate documents. Other responsibilities included facilitating peer to peer discussion and feedback following a code event and attending two review meetings each year. Committee members were encouraged to use this educational work for tiered advancement in their respective fields. Feedback on cardiac arrest areas for improvement was critical in creating a more streamlined approach to cardiac arrest care as well as to improve AHA compliance. Creation of the multidisciplinary cardiac arrest committee allowed for more recruitment of more interested individuals across multiple PICU roles. Given the nature of the project dependent on feedback and education across disciplines, attendance at the cardiac arrest meetings was critical. Given robust multidisciplinary participation at monthly PICU M\&M conferences, cardiac arrest review meeting was added to the M\&M PICU conference agenda. This improved more attendance and participation in event discussions and feedback. Subjectively, we went from five to six participants at cardiac arrest review meetings to a full room with standing room only during M\&M conferences.

Despite development of a cardiac arrest committee, we continued to have difficulty with timely notification of PICU cardiac arrests. In order to review all relevant telemetry data during an arrest event, the cardiac arrest review committee needed to be notified within 24 hours of the arrest. After 48 hours, the telemetry data are removed from the bedside Philips monitors and cardiac rhythms, arterial line and pulse oximetry waveform data may not be retrievable. Cardiac arrest notifications were often greater than 48 hours after the event occurred, leading to increased number of arrest reviews with incomplete waveform and cardiac rhythm analysis. Therefore, our third PDSA cycle spanning from July 2017 to January 2018, involved a daily automated text message to the PICU charge nurse phone asking about cardiac arrests that occurred in the PICU over the past 24 hours. Based on a yes or no reply, a follow-up phone call was made to obtain patient information. This allowed us to capture all cardiac arrests occurring in the PICU within 24 hours of an event. In order to provide a more secure data collection platform, a REDCap database was also created to streamline the review process and allow for a secure centralised location for all data.

There was great interest in the creation of a nurse lead education committee around cardiac arrest events based on feedback from nursing at M\&M conference. We felt that expanding the cardiac arrest review committee was critical in providing education around AHA guidelines and other interventions suggested during review committee meetings. During our fourth PDSA cycle, from January 2018 to July 2018, we created a nurse led Cardiac Arrest Education Committee consisting of six to eight nurses with at least 2 years of critical care experience who worked a variety of day and night shifts. Objectives of this committee included: leading peer-topeer postarrest debriefing sessions with bedside nursing staff to review specific resuscitative elements, attending at least two code committee reviews per year, and spearheading peer education around various cardiac arrest topics. Five cardiac arrest education topics were chosen during the first year including: (1) getting to know your code cart, (2) how to draw up code medications, (3) CPR basics including rate, depth and hand placement, (4) CPR during ECMO deployment and (5) rhythm recognition and interventions. These topics were chosen by the nursing committee members based on feedback from the prior year's cardiac arrest review meetings. An education bulletin board was placed in the break room of the PICU and each staff member was required to sign a sheet stating they read through the education materials. In addition, hands on simulations were led by the PICU fellows/APPs during both day and night shifts for relevant topic areas.

We continue to review all cardiac arrests occurring in the PICU and present data at our monthly multidisciplinary M\&M conference. Through sustained quality improvement efforts, we hope to improve our cardiac arrest outcomes over the next few years. Eventually, we hope to reduce the number of paediatric cardiac arrests in the PICU through early recognition of at-risk patients and timely intervention. 


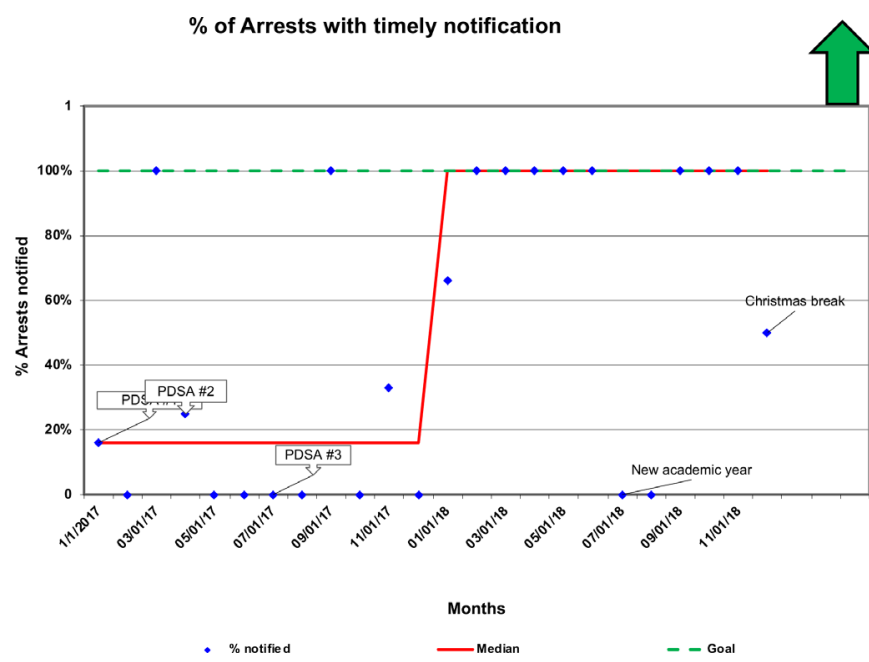

Figure 1 Statistical Process Control (SPC) run chart: percentage of cardiac arrest with timely notification by month from January 2017 to December 2018. PDSA, Plan-DoStudy-Act.

\section{RESULTS}

The primary aim of this project was to establish a multidisciplinary comprehensive cardiac arrest review process. This process was successfully implemented over a 2-year period, as evidenced by the creation of a standardised approach towards cardiac arrest in our PICU. Additional successes include, integration of the cardiac arrest review meeting with the monthly M\&M conference to optimise attendance, formation of a Nursing Cardiac Arrest Education Committee, along with multidisciplinary staff engagement in the initiative.

An Statistical Process Control (SPC) run chart was created to track percent of cardiac arrest with timely notification allowing for complete review (figure 1). Notifications of arrests were tracked monthly from 2017 to 2018. Percentage of notifications within a timely manner allowing for complete reviews of the cardiac arrest increased after initiation of a daily text message system. Notifications were near $100 \%$ after the daily text message system became automated. The decreased in notification percentage in July was attributed to new residents, fellows and nursing staff starting who were not aware of the cardiac review process. After reeducation, percentage of timely notification returned to $100 \%$. In December 2018, there was another decrease in timely notification to $50 \%$ due to holiday break and limited staff available to review cardiac arrest within 48 hours of notification.

Over the 2-year period, approximately 2\% of PICU admissions had a cardiac arrest, which is consistent with national data. ${ }^{12}$ There were a higher number of cardiac arrest events than patients because five patients in 2017 and two patients in 2018 had multiple cardiac arrests during their index PICU stay. The majority (56\%) of arrests were attributed to an underlying respiratory cause. In 2018 , we found that $52 \%$ of our arrests were pulseless electrical activity and $43 \%$ were bradycardic arrests; also consistent with published national data. ${ }^{12}$
Of the six $(27 \%)$ cardiac arrests with complete reviews in 2017, only $17 \%$ followed AHA guidelines for compression rate, depth and less than $10 \mathrm{~s}$ pauses during resuscitation. In 2018, these metrics improved drastically, with $76 \%$ of resuscitations noted to be compliant with AHA guidelines. Only $4 \%$ of cardiac arrests required ECPR between 2017 and 2018. Of the cardiac arrests that were reviewed by the committee, survival to discharge was $47 \%$ in 2017 and increased to $63 \%$ in 2018 with similar percentage of PICU patients having a cardiac arrest between the 2 years (1.6\%vs $2.0 \%)$. ROSC was similar between the 2 years with time to ROSC being slightly shorter in 2018 (5 min vs $3 \mathrm{~min})$. The acuity of illness and risk of mortality of patients admitted to the PICU did not significantly differ between the 2years (mortality index, case mix index 2017: 1.41, 2.71, 2018: 1.16, 2.53).

Our PICU codes per 1000 patient-days were similar between the 2 years at 4.7 and 4.5 . The average days between cardiac arrest events over the 2 years were 16.5, with wide month-to-month variation (table 2 ).

There was not an increase in the number of cardiac arrest events between 2017 and 2018, and no indication that patients were receiving CPR without meeting criteria. There was no impact of other PICU educational initiatives or time taken away from other M\&M discussions.

\section{CONCLUSIONS/LESSONS/LIMITATIONS}

The primary aim of this project was to create a multidisciplinary process to review cardiac arrests and implement a programme to improve the quality of CPR. The multidisciplinary approach was critical to our success. Involvement of attending physicians, fellows, nurses, respiratory therapists and leadership created an engaged and invested working group. Key lessons learnt included the importance of incorporating a new process into existing workflow and having early involvement of all stakeholders.

We also sought to increase compliance with AHA guidelines during CPR events. Our AHA compliance increased from $17 \%$ to $76 \%$ from 2017 to 2018 . It is difficult to draw conclusions from this as $73 \%$ of cardiac arrest had incomplete reviews in 2017. However, we feel that the creation of a comprehensive cardiac arrest review process with multi-disciplinary feedback could have led to increased AHA guideline compliance.

Despite the initial enthusiasm for the project, we identified several issues with reporting and reviewing cardiac arrests in a timely manner. Early on, several arrests were not reported, and the bedside telemetry was unable to be reviewed. In retrospect, implementing an automated daily text messaging system to the charge nurse sooner would have enabled timely reporting for this novel programme in our unit. Identifying both nursing leadership and respiratory therapy leadership earlier in the process would have also helped with staff investment and continued staff education.

This project highlighted several barriers to optimal team performance during resuscitations which could have contributed to the increase in AHA compliance during 
Table 2 Characteristics of paediatric cardiac arrests in the PICU in 2017 and 2018

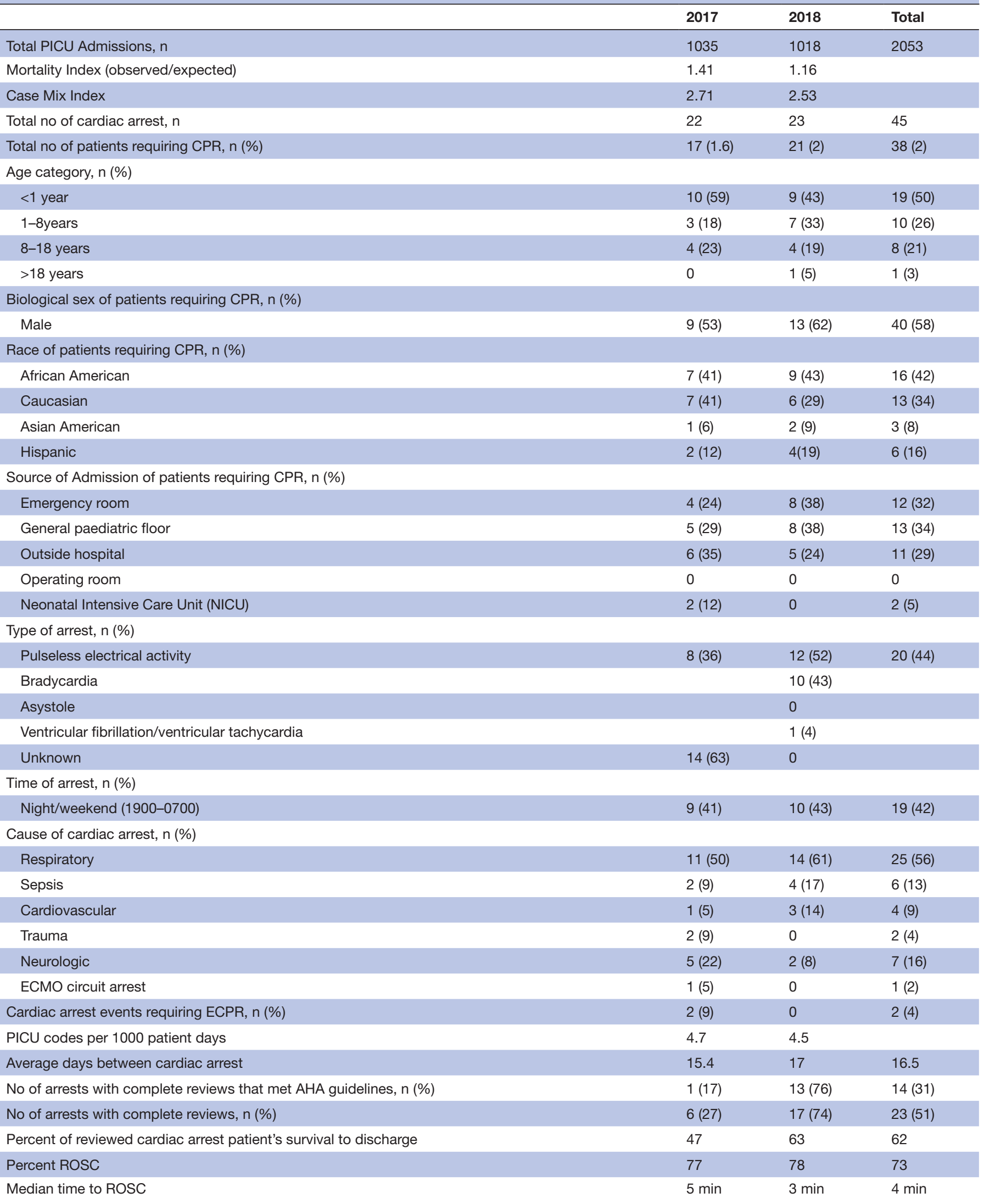

Patient race, gender and source of admission is expressed as a percentage of the total number of patients who required CPR to give a broader view of our PICU population.

AHA, American Heart Association; CPR, cardiopulmonary resuscitation ; ECMO, extracorporeal membrane oxygenation; PICU, paediatric intensive care unit; ROSC, return of spontaneous circulation. 
CPR between 2017 and 2018. Items as simple as changing the notification system for RT supervisor and PICU attendings of an in-unit code allowed for automatic notification of key team members. Other improvements attributed to our cardiac arrest initiative include development of an identification system for high-risk airways and consistent identification of the code leaders during resuscitation attempts.

While this project clearly improved our processes, there are several limitations. First, the lack of baseline data prior to our interventions limits our ability to fully demonstrate the impact of this initiative. Variability of fellow/APP staffing along with Registered Nurse (RN) and Respiratory Therapist (RT) staff turnover made it challenging to keep team members abreast of initiatives coming out of the code committee. Some of the early data collected were incomplete or not collected at all due to lack of communication. Missing $73 \%$ of our 2017 cardiac arrests prevented any meaningful clinical inferences to be drawn. Better notification and data capture would have enabled a more robust comparison between the two study years.

Cardiac arrest reviews are now an integral part of our PICU's M\&M conference ensuring that the reviews process is sustainable and will continue to occur on a monthly basis. Each academic year, a new fellow representative will join the cardiac arrest review committee, so that a minimum of 3 fellows (one per year) will be part of the review process, ensuring sustainable fellow presence. A paediatric intensivist faculty mentor will continue to oversee the cardiac reviews and provides mentorship to fellows. Next steps include continued data collection and tracking, understanding risk factors for cardiac arrest in a medical-surgical PICU, identification of pre-arrest trends which could hopefully prevent cardiac arrest events from occurring.

In conclusion, our cardiac arrest review and education programme allows for comprehensive multidisciplinary review of individual events, informs quality improvement initiatives, ensures consistent high-quality CPR and will hopefully lead to improved patient outcomes.

Correction notice This article has been corrected since it first published. The provenance and peer review statement has been included.

Acknowledgements Steven Hepditch RT. Steven was instrumental in providing large scale education for RT staff and helped with peer-to-peer feedback after cardiac arrests. Heather Harrison RN, is the nurse manager of the PICU at Duke. She provided support and peer to peer feedback to the nursing staff. She created the code corner to enable a centralised location of code debriefing forms.

Contributors All persons who meet authorship criteria are listed as authors, and all authors certify that they have participated sufficiently in the work to take public responsibility for the content, including participation in the concept, design, analysis, writing or revision of the manuscript. Furthermore, each author certifies that this material or similar material has not been and will not be submitted to or published in any other publication before its appearance in BMJ: Open Quality. CMR was involved in planning, conducting cardiac arrest reviews and presentations, recruitment of cardiac arrest team members, and the primary author of this manuscript. MP was involved in planning and expansion of the cardiac arrest review process, conducting cardiac arrest reviews and presentations, recruitment of cardiac arrest team members and contributing author to this manuscript. ER-A was involved with the planning, conducted cardiac arrest reviews, presenting reviews at multiple conferences and is a contributing author to this manuscript. $\mathrm{KJ}$ generated the concept of the cardiac review committee and helped initiate this review process in the PICU, she is also a contributing author for this manuscript. DAT is a contributing author for this manuscript and an active participant in the cardiac review meetings. SK was the mentor for the corresponding author, CMR. He was involved with the planning and implementation of the cardiac review process in the PICU. He is the senior author of this manuscript.

Funding The authors have not declared a specific grant for this research from any funding agency in the public, commercial or not-for-profit sectors.

Competing interests None declared.

Patient consent for publication Not required.

Provenance and peer review Not commissioned; externally peer reviewed.

Data availability statement All data relevant to the study are included in the article or uploaded as a supplimentary information.

Supplemental material This content has been supplied by the author(s). It has not been vetted by BMJ Publishing Group Limited (BMJ) and may not have been peer-reviewed. Any opinions or recommendations discussed are solely those of the author(s) and are not endorsed by BMJ. BMJ disclaims all liability and responsibility arising from any reliance placed on the content. Where the content includes any translated material, BMJ does not warrant the accuracy and reliability of the translations (including but not limited to local regulations, clinical guidelines, terminology, drug names and drug dosages), and is not responsible for any error and/or omissions arising from translation and adaptation or otherwise.

Open access This is an open access article distributed in accordance with the Creative Commons Attribution Non Commercial (CC BY-NC 4.0) license, which permits others to distribute, remix, adapt, build upon this work non-commercially, and license their derivative works on different terms, provided the original work is properly cited, appropriate credit is given, any changes made indicated, and the use is non-commercial. See: http://creativecommons.org/licenses/by-nc/4.0/.

ORCID iD

Candice M Ray http://orcid.org/0000-0002-7268-4566

\section{REFERENCES}

1 Berg RA, Nadkarni VM, Clark AE, et al. Incidence and outcomes of cardiopulmonary resuscitation in PICUs. Crit Care Med 2016;44:798-808.

2 Bhanji F, Topjian AA, Nadkarni VM, et al. Survival rates following pediatric in-hospital cardiac arrests during nights and weekends. JAMA Pediatr 2017;171:39-45.

3 Wolfe H, Zebuhr C, Topjian AA, et al. Interdisciplinary ICU cardiac arrest Debriefing improves survival outcomes*. Crit Care Med 2014;42:1688-95.

4 Sutton RM, Niles D, French B, et al. First quantitative analysis of cardiopulmonary resuscitation quality during in-hospital cardiac arrests of young children. Resuscitation 2014;85:70-4.

5 Smaha LA, American Heart Association. The American heart association get with the guidelines program. Am Heart $J$ 2004;148:S46-8.

6 Wolfe HA, Morgan RW, Zhang B, et al. Deviations from AHA guidelines during pediatric cardiopulmonary resuscitation are associated with decreased event survival. Resuscitation 2020;149:89-99.

7 Hunt EA, Jeffers J, McNamara L, et al. Improved Cardiopulmonary Resuscitation Performance With CODE ACES ${ }^{2}$ : A Resuscitation Quality Bundle. J Am Heart Assoc 2018;7:e009860.

8 Zebuhr C, Sutton RM, Morrison W, et al. Evaluation of quantitative Debriefing after pediatric cardiac arrest. Resuscitation 2012;83:1124-8.

9 Hamilton R. Nurses' knowledge and skill retention following cardiopulmonary resuscitation training: a review of the literature. $J$ Adv Nurs 2005;51:288-97.

10 Semeraro F, Signore L, Cerchiari EL. Retention of CPR performance in anaesthetists. Resuscitation 2006;68:101-8.

11 Sweberg T, Sen AI, Mullan PC, et al. Description of hot debriefings after in-hospital cardiac arrests in an international pediatric quality improvement collaborative. Resuscitation 2018;128:181-7.

12 Fanning RM, Gaba DM. The role of Debriefing in simulation-based learning. Simul Healthc 2007;2:115-25.

13 Kronick SL, Kurz MC, Lin S, et al. Part 4: systems of care and continuous quality improvement: 2015 American heart association guidelines update for cardiopulmonary resuscitation and emergency cardiovascular care. Circulation 2015;132:S397-413. 